BULLETIN OF THE

AMERICAN MATHEMATICAL SOCIETY

Volume 80, Number 1, January 1974

\title{
PERSISTENT MANIFOLDS ARE NORMALLY HYPERBOLIC
}

\author{
BY RICARDO MAN̄E
}

Communicated by M. H. Protter, June 29, 1973

Let $M$ be a compact, smooth, boundaryless manifold, and let $\operatorname{Diff}^{1}(M)$ be the group of $C^{\mathbf{1}}$-diffeomorphisms of $M$ endowed with the $C^{\mathbf{1}}$ topology. If $f \in \operatorname{Diff}^{1}(M)$, a compact invariant manifold of $f, V$, is a $C^{1}$ boundaryless submanifold of $M$, such that $f(V)=V$. In [1] and [2], M. Hirsch, C. Pugh and $M$. Shub proved that a normally hyperbolic compact invariant manifold (Definition 1 below) is persistent (Definition 2 below). The main purpose of this note is to announce the proof of the converse of this theorem.

\section{The main theorem.}

Definition 1. If $f \in \operatorname{Diff}^{1}(M)$, a compact invariant manifold of $f$, $V$, is normally hyperbolic if the tangent bundle of $M$ restricted to $V$, $T M / V$, has a splitting $T M / V=T V \oplus N^{s} V \oplus N^{u} V$ where $T V$ is the tangent bundle of $V$, and $N^{s} V, N^{u} V$, are $T f$-invariant subbundles of $T M / V$ such that there exist constants $K>0,0<\lambda<1$ satisfying

$$
\begin{gathered}
\left\|(T f)_{x}^{n} /\left(N^{s} V\right)_{x}\right\| \leqq K \lambda^{n}, \\
\left\|(T f)_{x}^{-n} /\left(N^{u} V\right)_{x}\right\| \leqq K \lambda^{n}, \\
\left\|(T f)_{x}^{n} /\left(N^{s} V\right)_{x}\right\|\left\|(T f)_{f^{n}(x)}^{-n} /(T V)_{f^{n}(x)}\right\| \leqq K \lambda^{n}, \\
\left\|(T f)_{x}^{-n} /\left(N^{u} V\right)_{x}\right\|\left\|(T f)_{f^{-n}(x)}^{n} /(T V)_{f^{-n}(x)}\right\| \leqq K \lambda^{n},
\end{gathered}
$$

for all $x \in V, n \in Z^{+}$.

Definition 2. If $f \in \operatorname{Diff}^{1}(M)$, a compact invariant manifold of $f$, $V$, is persistent if there exist a neighborhood $\mathscr{U}$ of $f$ in $\operatorname{Diff}^{1}(M)$ and a neighborhood $U$ of $V$ in $M$ such that:

(a) For all $g \in \mathscr{U}, V_{g}=\bigcap_{n \in Z} g^{n}(U)$ is a $C^{1}$ boundaryless submanifold of $M$ and $V_{f}=V$.

(b) If $g$ is $C^{1}$ near to $f, V_{g}$ is $C^{1}$ near to $V_{f}=V$.

AMS (MOS) subject classifications (1970). Primary 58F10, 58F15.

${ }^{1}$ The results announced here are part of the author's doctoral thesis at IMPA under the guidance of J. Palis. 
THEOREM. A compact invariant manifold $V$ of a diffeomorphism is persistent if and only if $V$ is normally hyperbolic.

As we observed above, the fact that normally hyperbolicity implies persistence is already known.

2. Other results. In the course of the proof of the main theorem the following situation arises frequently.

Definition. Let $K$ be a compact space, $N K$ a vector bundle over $K$ with a Finsler structure $\|\cdot\|$, and $N f$ an isomorphism of $N K$ covering $f$. We say that $K$ is $N f$-isolated in $N K$ if for all $x \in K, v \in(N K)_{x}, v \neq 0$, the set $\left\{\left\|(N f)_{x}^{n} v\right\| \mid n \in Z\right\}$ is not bounded. If $f \in \operatorname{Diff}^{1}(M)$, we say that it is a quasi-Anosov diffeomorphism if $M$ is $T f$-isolated in $T M$.

The following question is natural: Does quasi-Anosov imply Anosov? A partial answer is given by the following proposition.

Theorem. A quasi-Anosov diffeomorphism satisfies Axiom $\mathrm{A}$ and the no cycle condition. It is Anosov if and only if it is structurally stable, or, if and only if the dimensions of the stable manifolds of the periodic points are equal.

The following result relates this problem to a situation studied by Hirsch in [5].

Proposition. If $f$ is a quasi-Anosov diffeomorphism of $M$, there exist a manifold $N$, a $C^{\infty}$ embedding $i: M \rightarrow N$ and a $C^{1}$ diffeomorphism $g$ of $N$ such that $g \circ i=i \circ f$ and $i(M)$ is a hyperbolic set for $g$ [4]. Moreover:

$$
\operatorname{dim} M=\operatorname{dim} N+\left(\max _{x \in \operatorname{Per}(f)} \operatorname{dim} W^{u}(x)-\min _{x \in \operatorname{Per}(f)} W^{s}(x)\right),
$$

where $W^{s}(x)$ and $W^{u}(x)$ are the stable and unstable manifolds of $x$.

\section{REFERENCES}

1. M. Hirsch, C. Pugh and M. Shub, Invariant manifolds, (to appear).

2. - Invariant manifolds, Bull. Amer. Math. Soc. 76 (1970), 1015-1019. MR 45 \#1188.

3. S. Smale, Differentiable dynamical systems, Bull. Amer. Math. Soc. 73 (1967), 747-817. MR 37 \#3598.

4. M. Hirsch, Invariant subsets of hyperbolic sets, Symposium of Differential Equations and Dynamical Systems, Lectures Notes in Math., vol. 206, Springer-Verlag, Berlin and New York, 1971.

Instituto de Matematica Pura e Aplicada (IMPA), Rio de Janeiro, Brazil 